\title{
Isotopic fractionation of carbonyl sulfide in the atmosphere: Implications for the source of background stratospheric sulfate aerosol
}

\author{
Fok-Yan T. Leung, Agustín J. Colussi, and Michael R. Hoffmann \\ W.M. Keck Laboratories, California Institute of Technology, Pasadena, CA, USA
}

Geoffrey C. Toon

Jet Propulsion Laboratory, California Institute of Technology, Pasadena, CA, USA

Received 21 August 2001; revised 26 September 2001; accepted 27 September 2001; published 30 May 2002.

[1] In order to assess the contribution of carbonyl sulfide to stratospheric sulfate aerosol (SSA), we examined the stratospheric $\mathrm{OC}^{34} \mathrm{~S}$ and $\mathrm{OC}^{32} \mathrm{~S}$ concentration profiles in the infrared limbtransmittance spectra acquired by the JPL MkIV instrument. We found that $\mathrm{OC}^{34} \mathrm{~S}$ is preferentially depleted by solar photolysis. The derived ${ }^{34}$ S enrichment factor: $\varepsilon=+73.8 \pm 8.6 \%$, in conjunction with literature values of $\delta^{34} \mathrm{~S} \sim+11 \%$ for tropospheric OCS, and a $\sim$ $10 \%$ net processing of the OCS transported upwardly into the stratosphere, suggests that aerosol sulfate proceeding from OCS should be highly enriched in $\delta^{34} \mathrm{~S} \sim 80 \%$, comparing our prediction with previous reports of $\delta^{34} \mathrm{~S} \sim+2.6 \%$ for background SSA, we infer either that OCS is a minor contributor to SSA or that current views about its ${ }^{34} \mathrm{~S}$-abundance and atmospheric circulation are seriously flawed. INDEX TERMS: 0340 Atmospheric Composition And Structure: Middle atmosphere-composition and chemistry

\section{Introduction}

[2] The stratospheric sulfate aerosol layer, a persistent, optically thin layer of concentrated sulfuric acid microdroplets that extends upwards from the tropopause, regulates the albedo of Earth's atmosphere and plays a crucial role in the chemistry of the lower stratosphere. SSA particles catalyze heterogeneous reactions that convert $\mathrm{NO}_{2}$ into nitric acid, reducing $\mathrm{NO}_{\mathrm{x}}$ and hence indirectly increasing the amount of available free chlorine [Hanson et al., 1994; McElroy et al., 1992].

[3] Since the early observations and experiments on stratospheric aerosol [Junge et al., 1961], there has been considerable debate regarding the sources of SSA sulfur. Volcanic eruptions represent major perturbations to atmospheric sulfur, but have a passing effect on the SSA layer [Sedlacek et al., 1983]. It has been conjectured that carbonyl sulfide, a relatively inert gas, could be the long term source of the background layer [Crutzen, 1976; Turco et al., 1980]. Although OCS is a highly plausible precursor of background SSA, it is difficult to assess the extent of its contribution given the uncertainties associated with global OCS sources and sinks [Chin and Davis, 1995; Watts, 2000]. Actually, the sulfur influx from tropospheric OCS may be is insufficient to maintain steady state SSA levels [Chin and Davis, 1995; Kjellstrom, 1998]. Current models tend to underpredict aerosol concentrations under $20 \mathrm{~km}$, but overestimate them at higher altitudes [Hofmann, 1991]. The known pathways for OCS destruction in the gas phase are photodissociation, and to a lesser extent, oxidation by $\mathrm{O}$ and $\mathrm{OH}$ radicals [Chin and Davis,
1995]. Recent work ruled out the possible importance of heterogeneous oxidation of OCS by $\mathrm{H}_{2} \mathrm{O}_{2}$ [Dalleska et al., 2000]. It has been suggested that additional sulfur sources may contribute significantly to SSA in the lower stratosphere. The $\mathrm{SO}_{2}$ uplifted from the lower troposphere by deep convective events at the tropics could be a major contributor to SSA [Weisenstein et al., 1997].

[4] An aftermath approach to this problem involves searching for isotopic signatures. The isotopic composition of stratospheric sulfate is inextricably linked to the isotopic composition of its sources, and to the mechanism and extent of their oxidation [Krouse and Grinenko, 1991; Rahn and Wahlen, 1997; Rahn and Wahlen, 2000]. Data on the isotopic composition of atmospheric OCS and of SSA particles would constrain the OCS loss mechanisms and its contribution to background stratospheric sulfate [Goldman et al., 2000]. In this work we use the vertical profile of both $\mathrm{OC}^{34} \mathrm{~S}$ and $\mathrm{OC}^{32} \mathrm{~S}$ isotopologues from the high resolution IR spectra previously used by Griffith et al. in their study of $\mathrm{N}_{2} \mathrm{O}$ isotopomers [Griffith et al., 2000], plus additional spectra recorded in the year 2000, to establish the sign and magnitude of sulfur isotope fractionation resulting from the stratospheric processing of OCS. Combining this information with previous data on the sulfur isotopic ratio in SSA particles, and with recent estimates of the tropospheric OCS isotopic composition, we were able to arrive at an upper limit for the contribution of OCS to the SSA.

\section{Experimental}

[5] Isotopologues of OCS can be quantified on the basis of their distinctive rovibrational band spectra in the near infrared region. In this work, we analyzed nine sets of high resolution infrared solar occultation spectra recorded by the MkIV instrument on balloon flights launched from Fort Summer, New Mexico (34N), Esrange, Sweden (68N), Fairbanks, Alaska $(64 \mathrm{~N})$, Lynn Lake, Manitoba $(57 \mathrm{~N})$, and Daggett, California (34N) between 1992 and 2000 [Toon et al., 1999; Toon, 1991]. For each balloon flight, spectra were typically acquired at tangent altitudes between about $5 \mathrm{~km}$ and $40 \mathrm{~km}$ altitude, at 1 to $3 \mathrm{~km}$ intervals.

[6] The spectral region between 2041 and $2077 \mathrm{~cm}^{-1}$, centered on the $\nu_{3}$ asymmetric $\mathrm{OC}^{32} \mathrm{~S}$ stretch at $2054 \mathrm{~cm}^{-1}$, was analyzed using the GFIT program, which involves a least squares regression of known spectral library data to the acquired spectra. A sixty 1-km layer model of the atmosphere was used. Spectroscopic parameters from the HITRAN 1996 were supplemented by recent measurements of OCS [Strugariu et al., 1998] and of isotopic $\mathrm{O}_{3}$ absorption bands [Barbe, 2001]. The 50 $\mathrm{cm}^{-1}$ region centered on the weaker $\nu_{1}$ OCS band at $850 \mathrm{~cm}^{-1}$ was also examined to test the robustness of the analysis, the 


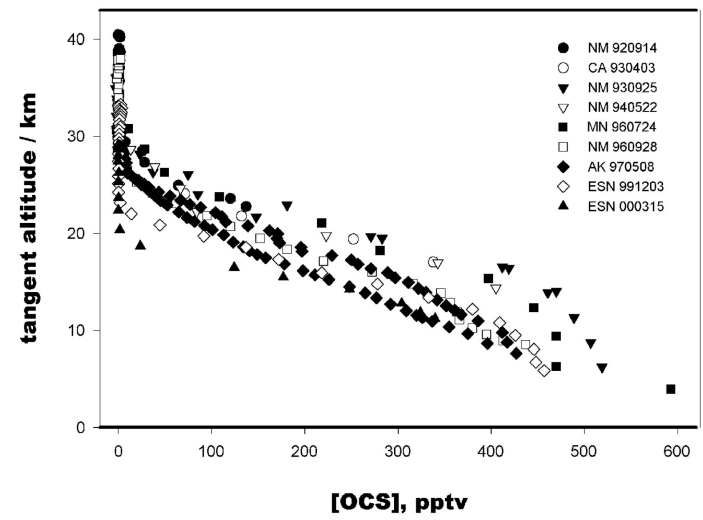

Figure 1. Tangent height vertical profiles for total OCS (i.e.: $\mathrm{OC}^{34} \mathrm{~S}+\mathrm{OC}^{32} \mathrm{~S}$ ) partial pressures. The locations and launch dates of the missions are identified in the legend as: $\mathrm{MN}=$ Manitoba, Canada; ESN = Esrange, Sweden; ALK = Alaska, NM = New Mexico; and CA = California, USA, and YY/MM/DD time format, respectively. We have combined profiles that were measured during the same flight.

results being in agreement with those from the 2041-2077 $\mathrm{cm}^{-1}$ region.

\section{Results and Discussion}

[7] The ${ }^{34} \mathrm{~S}$ relative excess in OCS samples $\delta^{34} \mathrm{~S}$, is defined as:

$$
\delta^{34} \mathrm{~S}=1000 \times\left[\frac{\left({ }^{34} \mathrm{~S} /{ }^{32} \mathrm{~S}\right)_{\text {sample }}}{\left({ }^{34} \mathrm{~S} /{ }^{32} \mathrm{~S}\right)_{\text {standard }}}-1\right]
$$

The standard is meteoritic Cañon Diablo troilite. Because of the relatively large natural abundance of ${ }^{34} \mathrm{~S}(4.2 \%)$ compared to those of the heavier isotopes of carbon and oxygen, $\mathrm{OC}^{34} \mathrm{~S}$ and $\mathrm{OC}^{32} \mathrm{~S}$ are the only relevant isotopologues. Therefore, the analysis of the spectra of OCS isotopologues is simpler than in the case of $\mathrm{N}_{2} \mathrm{O}$, which has $2{ }^{15} \mathrm{~N}$ positional isotopomers, ${ }^{15} \mathrm{~N}^{14} \mathrm{NO}$ and ${ }^{14} \mathrm{~N}^{15} \mathrm{NO}$, in addition to the parent ${ }^{14} \mathrm{~N}^{14} \mathrm{NO}$ isotopologue.

[8] Isotope fractionation will result during the photolysis of OCS if the ratio of actinic light absorbances differed from the relative isotopologue concentrations [Miller and Yung, 2000]:

$$
\begin{aligned}
& O C^{34} S+h v \stackrel{k_{2}}{\longrightarrow}{ }^{34} S+C O \\
& O C^{32} S+h v \stackrel{k_{3}}{\longrightarrow}{ }^{32} S+C O
\end{aligned}
$$

$\delta^{34} \mathrm{~S}$ is usually reported for local concentrations at a particular altitude, rather than for slant column abundances. However, to avoid the ambiguities associated with the retrieval of local concentrations from slant column abundances, we based our analysis on raw slant column abundances. Since slant column abundances are strongly weighted to the tangent altitudes, they give a good approximation to the conventionally defined $\delta^{34} \mathrm{~S}$ at that tangent altitude, especially for gases like OCS and $\mathrm{N}_{2} \mathrm{O}$ whose volume mixing ratio decreases with altitude [Griffith et al., 2000]. The volume mixing ratio (vmr) profile for OCS, plotted against tangent altitude, is shown in Figure 1. If the ratio $\mathrm{k}_{2} / \mathrm{k}_{3}$ were independent of altitude, the isotopic enrichment of OCS could be dealt with as a Rayleigh distillation [Griffith et al., 2000; Rahn and Wahlen, 1997]. For OCS at any altitude, the

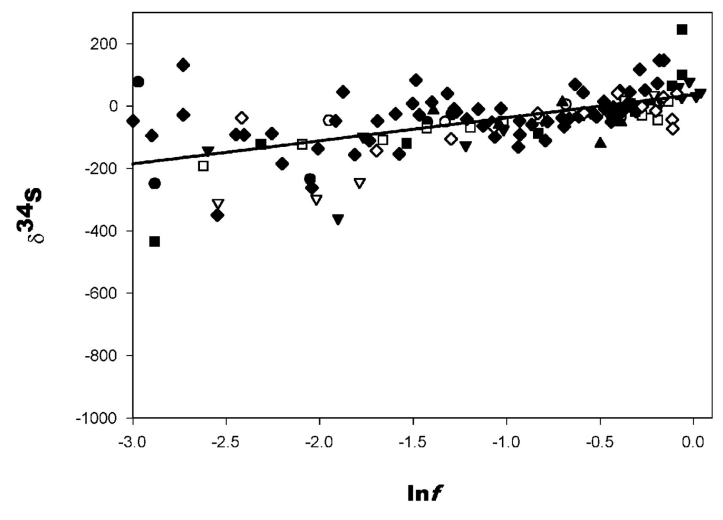

Figure 2. ${ }^{34} \mathrm{~S}$ abundance in stratospheric OCS as function of the unreacted fraction. The mixing ratio of OCS in the troposphere was assumed to be 0.5 . Flights are identified as in Figure 1.

isotopic ratio $\mathrm{R}$ would be then related to the tropospheric isotopic value $R_{o}$ by:

$$
R=R_{0} f^{\alpha-1}
$$

where $f=[\mathrm{OCS}]_{\mathrm{z}} /[\mathrm{OCS}]_{\text {troposphere }}$ is the fraction of unphotolyzed OCS, and $\alpha=\mathrm{k}_{2} / \mathrm{k}_{3}$ the ratio of photolysis rates for the heavier to the lighter isotopomer. This leads, as a good approximation, to

$$
\delta=\delta_{0}+\varepsilon \ln f
$$

where $\varepsilon=100 \times(\alpha-1)$ is the enrichment factor. The apparent linearity of the Rayleigh plot of $\delta^{34} \mathrm{~S}$ vs. $\ln f$ (Figure 2) confirms our assumptions. The enrichment factor, $\varepsilon$, is given by the slope of the Rayleigh plot. Deviations from linearity would have arisen from the involvement of additional loss mechanism, or from the dependence of mass transport parameters on altitude.

[9] The present analysis is very similar to that performed by Griffith et al, for $\mathrm{N}_{2} \mathrm{O}$. However, the natural abundance of OCS at the tropopause is $\sim 0.5 \mathrm{ppbv}$, compared to $315 \mathrm{ppbv}$ for $\mathrm{N}_{2} \mathrm{O}$. This explains at least in part the greater degree of scatter in the OCS Rayleigh plot compared to that for $\mathrm{N}_{2} \mathrm{O}$ [Griffith et al., 2000]. Uncertainties reported by the spectral fitting algorithm (not reported) are even larger. These uncertainties arise from systematic errors due to inadequacies in the spectroscopic library, which are independent of altitude. However, systematic errors affect $\mathrm{OC}^{32} \mathrm{~S}$ and $\mathrm{OC}^{34} \mathrm{~S}$ measurements to similar extents and, therefore, should not modify the calculated sulfur isotopic enrichment factor, $\varepsilon$. However, as noted in Griffith et al., the measured $\varepsilon$ 's are sensitive to errors that are not independent of altitude and/or absorption strength in the spectra, such as zero offsets, temperature profiles and assumed line broadening coefficients. Sensitivity analyses on these factors showed that the results are robust. Calculated $\delta^{34} \mathrm{~S}$ values are unreliable below $\sim 10 \mathrm{~km}$ because of the increasing atmospheric opacity, and above

Table 1. Calculated Enrichment factors

\begin{tabular}{lcc}
\hline \multicolumn{1}{c}{ Flight } & Enrichment Factor & Std. error \\
\hline April 1993, CA & 94.2 & 20.2 \\
September 1996, NM & 120.5 & 30 \\
May 1997, AK & 70.4 & 6.2 \\
July 1997, ALK & 59.1 & 13.6 \\
December 1999, ESN & 80.8 & 19 \\
Weighted average of above & 71.1 & 21.9 \\
Average of all flights & 73.8 & 8.6 \\
\hline
\end{tabular}




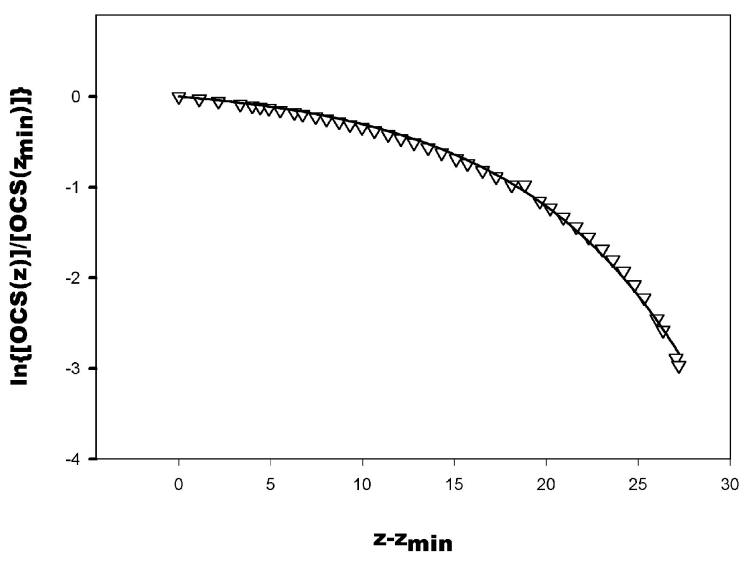

Figure 3. Normalized OCS concentration as a function of normalized altitude. $z_{\min }$ in this case is $7.6 \mathrm{~km}$. The solid line is a regression of equation 6 to experimental data. The data used was from the flight launched from Fairbanks Alaska in May, 1997 (AK 970508).

about $20 \mathrm{~km}$, where the $\mathrm{OC}^{34} \mathrm{~S}$ absorption lines become very weak in comparison with the spectral noise. They were therefore excluded from the Rayleigh plot analysis (Figure 2).

[10] Enrichment factors were calculated both using the entire dataset, which includes all flights, and using data from individual flights that contained sufficient data to perform meaningful statistical analyses. Regression parameters were derived by assigning individual points, which were weighted proportional to $1 /$ error $^{2}$ [Bevington, 1969]. The enrichment factor calculated by averaging the results from individual flights were also weighted as $1 /$ (standard error $)^{2}$. The results are reported in Table 1 . Despite the considerable variability between the values of individual missions, the enrichment factor calculated from the entire dataset is in good agreement with the weighted average of the $\varepsilon$ 's.

[11] The possible mechanisms of OCS destruction in the lower stratosphere include photolysis, which is believed to account for most of OCS processing, and oxidation by $\mathrm{O}$ or $\mathrm{OH}$ [Chin and Davis, 1995]. The relative importance of such processes varies with the concentrations of the oxidizing species and the opacity of the atmosphere, i.e., with altitude. The apparent enrichment factors might also vary with latitude and season. If the actual sulfur isotopic enrichment factors associated with the different processes were to differ significantly, Rayleigh plots could deviate from linearity. However, it is impossible to ascertain seasonal or latitudinal dependencies from the limited number of missions available. More accurate or more comprehensive data would be needed to expose such dependencies.

[12] It is possible to test the hypothesis that OCS photolysis by solar radiation screened by ozone is the major loss channel in the stratosphere from the local ozone concentrations retrieved from the same spectral dataset. The actinic radiation at the lower altitudes peaks at about $215 \mathrm{~nm}$, which lies about $10 \mathrm{~nm}$ below the absorption maximum of OCS at about $223 \mathrm{~nm}$ [Wu et al., 1999]. Combining the actinic photon flux filtered by the ozone column from the top of the column down to altitude $\mathrm{z}$ with the OCS mass balance, we obtain:

$$
\ln \frac{[O C S]_{z}}{[O C S]_{z \min }}=A\left\{1-\exp \left[\gamma\left(z-z_{\min }\right)\right]\right\}
$$

where A and $\gamma$ are combinations of spectral and transport parameters. Equation 6 provides a realistic representation of the experimental [OCS] vs $\mathrm{z}$ data (Figure 3). It is important to note that observations were made from a balloon flight flying at high latitudes and outside the polar vortex, but before it has disintegrated. The [OCS] vertical profile is therefore not affected by latitudinal transport, the effects of which are not considered in the above analysis. This restriction does not detract from the conclusion that OCS photodissociation is the major stratospheric loss channel for OCS.

[13] The observed enrichment factor has a large positive value, in contrast to the large negative value measured for stratospheric $\mathrm{N}_{2} \mathrm{O}$ [Griffith et al., 2000]. The contrasting behaviors arise from the fact that the actinic radiation in both cases is centered about $215 \mathrm{~nm}$, which lies to the blue of the OCS absorption maximum at $223 \mathrm{~nm}$, but to the red of that for $\mathrm{N}_{2} \mathrm{O}$ at $183 \mathrm{~nm}$. Therefore, according to the recent analysis of the UV absorption spectral shifts induced by isotope substitution [Miller and Yung, 2000], the photolysis of $\mathrm{OC}^{34} \mathrm{~S}$, and ${ }^{14} \mathrm{~N}^{14} \mathrm{~N}$ should proceed at faster rates. Thus, the products of the photolysis of OCS should be enriched in ${ }^{34} \mathrm{~S}$, i.e, $\varepsilon>$ 0 , as observed. The theory, which is based on an equilibrium analysis of zero point energy effects, is qualitatively correct but could not actually predict $\varepsilon$ values in the case of $\mathrm{N}_{2} \mathrm{O}$ photolysis. The deficiency is apparently associated with dynamic effects related to the fact that these linear triatomics are actually bent in their excited states. Therefore, the light absorption from hot bands involving bending overtones of the ground states is disproportionately larger than their thermal populations as a result of the favorable FranckCondon factors, an effect that was not considered by Miller and Yung [Zhang et al., 2000].

\section{Implications}

[14] As noted previously, atmospheric OCS fluxes are rather poorly constrained by existing measurements. Based on known chemical and photochemical rates, Chin and Davis [1995] estimated that less than $10 \%$ of the OCS transported into the stratosphere is actually destroyed there. The remainder re-enters the troposphere. Based on this appraisal, our enrichment factor of $\varepsilon \sim+73 \%$, and Krouse and Grinenko's [1991] estimate of $\delta^{34} \mathrm{~S} \sim$ $11 \%$ for tropospheric OCS, we infer from mass balance considerations that any sulfate produced from OCS should have a $\delta^{34} \mathrm{~S}_{\text {sulfate }} \sim 80 \%$. [From $\delta^{34} \mathrm{~S}_{\text {sulfate }}(1-\mathrm{f})+\delta^{34} \mathrm{~S} \mathrm{f}=\delta_{0}{ }^{34} \mathrm{~S}$, where $\delta^{34} \mathrm{~S}=11 \%$ o $+73 \ln (0.9)$ Equation (5)]. In contrast, Castleman et al. [1974] found that SSA $\delta^{34} \mathrm{~S}_{\text {sulfate }} \sim 2.6 \%$ in background. This large discrepancy suggests that carbonyl sulfide would contribute negligibly to stratospheric aerosol during volcanically quiescent periods. In fact, assuming that $\delta^{34} \mathrm{~S}$ values for atmospheric $\mathrm{SO}_{2}$ and background aerosol are identical within $5 \%$, one can estimate that OCS contributes less than $6 \%$ to the SSA sulfur budget.

[15] It should be emphasized that the predicted $\delta^{34} \mathrm{~S} \sim 80 \%$ value for the product sulfate depends on the assumed stratospheric conversion of OCS via Eq. (5). Thus, the $\delta^{34} \mathrm{~S}$ for the resultant sulfate decreases from $\sim 84 \%$ at $f=0$, to the tropospheric value for OCS, $\delta^{34} \mathrm{~S} \sim 11 \%$ at $100 \%$ conversion. In other words, as $f$ increases, the discrepancy between predicted and observed $\delta^{34} S^{\prime}$ s for SSA converges to the difference between 2.6 and 11\%. Therefore, the discrepancy would be largely removed if Chin and Davis [1995] had significant underestimated the extent of OCS conversion. In that event, the discrepancy would ultimately be associated with the tentative estimates of $\delta^{34} \mathrm{~S}$ in tropospheric OCS made by Krouse and Grinenko [1991]. These caveats underscore the need for more accurate sulfur isotopic data, which are in principle experimentally accessible, to provide definitive clues regarding the origin of background stratospheric sulfate aerosols.

\section{Conclusions}

[16] From the analysis of high-resolution infrared spectra of the terrestrial atmosphere obtained by balloon flights spanning ten 
years, which cover various latitudes and seasons, we reach the following conclusions:

i) The apparent ${ }^{34} \mathrm{~S}$ enrichment factor for stratospheric processing of carbonyl sulfide is $\varepsilon=73 \pm 8 \%$.

ii) The vertical profile of OCS concentrations can be quantitatively accounted for by photolysis under solar radiation screened by the ozone layer.

iii) A positive $\varepsilon$ value is qualitatively consistent with an equilibrium analysis of zero point energy effects on the UV absorption spectrum of OCS.

iv) The measured enrichment factor implies that any sulfate derived from stratospheric OCS should be highly enriched in ${ }^{34} \mathrm{~S}$, at variance with the sulfur isotopic ratio observed in SSA particles.

v) Further work is necessary to close the gaps in our understanding of stratospheric sulfate aerosol and the carbonyl sulfide budget.

[17] Acknowledgments. Thanks to Prof. Yuk Yung (Caltech) for invaluable advice and for allowing us to use his group's facilities, and to Alain Barbe for providing the spectroscopic information on $\mathrm{O}_{3}$ isotopomers necessary for the spectral analysis.

\section{References}

Barbe, A., Personal Communication, 2001

Bevington, P. R., Data Reduction and Error Analysis for the Physical Sciences, 336 pp., McGraw-Hill, 1969.

Castleman, J. A. W., H. R. Munkelwitz, and B. Manowitz, Isotopic studies of the sulfur component of the stratospheric aerosol layer, Tellus, 26, $222-234,1974$

Chin, M., and D. D. Davis, A reanalysis of carbonyl sulfide as a source of stratospheric background sulfur aerosol, Journal of Geophysical Research, 100, 8993-9005, 1995.

Crutzen, P. J., The possible importance of CSO for the sulfate aerosol layer of the stratosphere, Geophysical Research Letters, 3(2), 73-76, 1976.

Dalleska, N. F., A. J. Colussi, A. M. Hyldahl, and M. R. Hoffmann, Rates and mechanism of carbonyl sulfide oxidation by peroxides in concentrated sulfuric acid, Journal of Physical Chemistry - A, 104(46), 10,794$10,796,2000$

Goldman, A., et al., Isotopic OCS from high-resolution balloon-borne and ground-based infrared absorption spectra, Journal of Quantitative Spectroscopy and Radiative Transfer, 67, 447-455, 2000.

Griffith, D. W. T., et al., Vertical profiles of nitrous oxide isotopomer fractionation measured in the stratosphere, Geophysical Research Letters, $27,2485-2488,2000$.

Hanson, D. R., A. R. Ravishankara, and S. Solomon, Heterogeneous reac- tions in sulfuric acid aerosols - a framework for model calculations, Journal of Geophysical Research, 99(D2), 3615-3629, 1994.

Hofmann, D. J., Aircraft sulphur emissions, Nature, 349, 659, 1991.

Junge, C. E., C. W. Chagnon, and J. E. Manson, Stratospheric Aerosols, Journal of Meteorology, 81-108, 1961.

Kjellstrom, E., A three dimensional global model study of carbonyl sulfide in the troposphere and the lower stratosphere, Journal of Atmospheric Chemistry, 29, 151-177, 1998

Krouse, H. R., and V. A. Grinenko, editors, Stable Isotopes: Natural and Anthropogenic sulphur in the Environment, Scope 43, 400 pp., John Wiley and Sons, 1991.

McElroy, M. B., R. J. Salawitch, and K. Minschwaner, The Changing Stratosphere, Planetary and Space Science, 40(2-3), 373-401, 1992.

Miller, C. E., and Y. L. Yung, Photo-induced isotopic fractionation, Journal of Geophysical Research, 105(D23), 29,039-29,051, 2000.

Rahn, T., and M. Wahlen, Stable isotope enrichment in stratospheric nitrous oxide, Science, 278, 1776-1778, 1997.

Rahn, T., and M. Wahlen, A reassessment of the global isotopic budget of atmospheric nitrous oxide, Global Biogeochemical Cycles, 14, 537-543, 2000

Sedlacek, W. A., et al., A decade of stratospheric sulfate measurements compared with observations of volcanic eruptions, Journal of Geophysical Research, 88, 3741-3776, 1983.

Strugariu, T., et al., Fourier transformation spectrscopy of O-18-enriched carbonyl sulfide form 1825 to $2700 \mathrm{~cm}-1$, Journal of Molecular Spectroscopy, 189(2), 206-219, 1998.

Toon, G., et al., Comparison of MkIV balloon and ER-2 aircraft measurements of atmospheric trace gases, Journal of Geophysical Research Atmopheres, 104(D21), 26,779-26,790, 1999.

Toon, G. C., The JPL MkIV interferometer, Optics and Photonics News, $19-21,1991$

Turco, R. P., et al., OCS, stratospheric aerosols and climate, Nature, 283, $283-286,1980$

Watts, S. F., The mass budgets of carbonyl sulfide, dimethyl sulfide, carbon disulfide and hydrogen sulfide, Atmospheric Environment, 34, 761-779, 2000 .

Weisenstein, D. K., et al., A two-dimensional model of sulfur species and aerosols, Journal of Geophysical Research, 102, 13,019-13,035, 1997.

Wu, C. Y. R., F. Z. Chen, and D. L. Judge, Temperature dependent photoabsorption cross sections of OCS in the 2000-2600 A region, J. Quant. Spectrosc. Radiat. Transfer, 61, 265-271, 1999.

Zhang, H., P. O. Wennberg, V. H. Wu, and G. A. Blake, Fractionation of ${ }^{14} \mathrm{~N}^{15} \mathrm{~N}^{16} \mathrm{O}$ and ${ }^{15} \mathrm{~N}^{14} \mathrm{~N}^{16} \mathrm{O}$ during photolysis at $213 \mathrm{~nm}$, Geophysical Research Letters, 27(16), 2481-2484, 2000.

A. J. Colussi, M. R. Hoffmann (corresponding author), and F. -Y. Leung, W. M. Keck Laboratories 138-78, California Institute of Technology, Pasadena, CA 91125, USA. (ajcoluss@its.caltech.edu; mrh@its.caltech.edu; fok-yan@its.caltech.edu)

G. C. Toon, Jet Propulsion Laboratory, California Institute of Technology, Pasadena, CA 91109, USA. (toon@mark4sun.jpl.nasa.gov) 